\title{
Влияние электрического поля на движущийся экситон в GaAs
}

\author{
( Д.К. Логинов, П.А. Белов, И.Я. Герловин, И.В. Игнатьев \\ Санкт-Петербургский государственный университет, Лаборатория оптики спина им. И.Н. Уральцева, \\ 198504 Санкт-Петербург, Петергоф, Россия \\ E-mail: loginov999@gmail.com
}

Поступила в Редакцию 12 апреля 2021 г.

В окончательной редакции 19 апреля 2021 г.

Принята к публикации 19 апреля 2021 г.

Выполнено моделирование интерференции поляритонных волн в широкой квантовой яме в присутствии внешнего однородного электрического поля. Показано, что в зависимости от значений приложенного поля эта интерференция изменяется с конструктивной на деструктивную. Этот процесс описывается линейным по волновому вектору членом в экситонном гамильтониане.

Ключевые слова: экситон, электрическое поле, квантовая яма, светоэкситонное взаимодействие.

DOI: 10.21883/FTP.2021.09.51294.24

\section{1. Введение}

В оптических спектрах квантовых ям (КЯ), ширина которых превосходит на порядок экситонный боровский радиус, можно наблюдать особенности отражения, связанные с уровнями размерного квантования движения экситона как целого [1-3]. Это позволяет исследовать состояния движения экситона с большим волновым вектором, $K \gg q$, где $q-$ волновой вектор падающего света. Оптические исследования гетероструктур с такими широкими КЯ, начатые во второй половине прошлого столетия [4-10], остаются актуальными вплоть до настоящего времени [11-17].

Особый интерес представляет возможность исследовать с помощью оптической спектроскопии влияние различных внешних возмущений на экситонные состояния с большим волновым вектором. Так, например, исследования экситонных спектров широких КЯ во внешнем магнитном поле показали ряд необычных эффектов, не наблюдавшихся ранее в объемных материалах. В геометрии Фарадея магнитное поле приводило к зависимости $g$-фактора экситона от волнового вектора [18-20], а в геометрии Фогта к эффективному увеличению массы экситона [21-24]. Также было установлено, что нетривиальные эффекты можно наблюдать в спектрах КЯ в поле механической деформации. При сжатии КЯ вдоль оси 4-го порядка должен наблюдаться эффект инверсии фазы спектральных осцилляций [25]. При сжатии КЯ вдоль оси 2-го порядка в оптических спектрах наблюдаются изменения, обусловленные эффективным сближением масс экситонов тяжелой и легкой дырок [26].

Влияние электрического поля на экситоны исследуется уже несколько десятилетий [27-34]. В большей части этих работ экситон в электрическом поле рассматривается либо в объемном кристалле [27-29], либо в относительно узких КЯ, размер которых примерно равен либо меньше экситонного боровского радиуса [30-32]. Движение центра масс в таких узких КЯ отсутствует.
Как следствие, спектроскопия как объемных кристаллов, так и узких КЯ не позволяет исследовать влияние электрического поля на состояния экситона с большим волновым вектором.

Эффекты электрического поля в широких КЯ к настоящему времени исследованы в меньшей степени. Авторами работ $[35,36]$ теоретически исследовано влияние электрического поля на светоэкситонное взаимодействие в широкой КЯ. В работе [37] предложена модель, описывающая инверсию фазы спектральных особенностей, связанных с уровнями размерного квантования экситона. Этот эффект аналогичен эффекту, предсказанному для действия одноосного сжатия в работе [25].

В работе [38] исследованы спектры электроотражения гетероструктуры с КЯ GaAs шириной 120 нм. В этих спектрах наблюдались особенности (осцилляции отражения), связанные с уровнями размерного квантования движения экситона. Внешнее электрическое поле $F$, приложенное под некоторым углом $\theta$ к оси роста структуры, приводило к уменьшению амплитуды осцилляций вплоть до полного нуля при некотором критическом значении $F_{c}$. Однако при дальнейшем увеличении поля осцилляции снова появлялись. Фаза этих осцилляций была инвертирована по сравнению с их фазой при $F<F_{c}$. Авторами работы [38] предложена модель, описывающая наблюдаемый эффект. Инверсия спектральных осцилляций описывается линейным по волновому вектору экситона, $K$, членом в экситонном гамильтониане, индуцированным компонентой поля, направленной в плоскости КЯ. В настоящей работе мы демонстрируем, что в достаточно сильном поле возможна многократная инверсия фазы поляритонных резонансов электроотражения.

\section{2. Модель}

Рассмотрим электроотражение от гетероструктуры, содержащей широкую КЯ GaAs/AlGaAs. Считаем, что гетероструктура состоит из внешнего барьерного слоя, 
слоя КЯ и полубесконечного слоя подложки. Для определенности будем считать, что свет, падающий по нормали на гетероструктуру, имеет левую циркулярную поляризацию. Предполагается, что ось роста гетероструктуры (ось $z$ ), вдоль которой происходит распространение света, совпадает с кристаллографическим направлением [001]. При дальнейшем изложении ось $z$ считается также осью квантования спиновых моментов фотона и экситона. Координатные оси $x$ и $y$ направлены вдоль осей 4-го порядка $[100]$ и [010] соответственно. Предполагается, что к гетероструктуре приложено однородное электрическое поле $\mathbf{F}$, вектор которого отклоняется от оси $z$ в плоскости $(z x)$.

Для анализа экситонного вклада в поляризацию среды используем модель интерференции экситоноподобных и фотоноподобных поляритонных волн [1-3]. Дисперсионные соотношения между энергией и волновым вектором этих волн можно установить из двух выражений для диэлектрической функции среды. Первое из них имеет вид [1-3]

$$
\varepsilon(\omega, K)=\frac{c^{2} K^{2}}{\omega^{2}}
$$

где $c-$ скорость света, $\omega-$ частота света. Второе выражение, учитывающее экситонный вклад в диэлектрическую функцию, в базисе циркулярно-поляризованных волн описывается выражением [38]

$$
\varepsilon_{ \pm}(\omega, K)=\varepsilon_{0}+\frac{\varepsilon_{0} \cdot \hbar \omega_{L T}(F)}{H_{ \pm}(F, K)-\hbar \omega+i \hbar \Gamma} .
$$

Здесь $\hbar \omega_{L T}(F)$ - продольно-поперечное расщепление экситона, $\hbar \Gamma-$ параметр затухания экситона. Энергия экситона $H_{ \pm}(G F, K)$ описывается выражением

$$
H_{ \pm}(F, K)=E_{g}-R+\frac{\hbar^{2} K^{2}}{2 M} \pm \lambda(F) \xi K .
$$

Здесь $R-$ экситонная энергия связи. Величина $M=m_{h}+m_{e}-$ масса экситона, в которой $m_{e}=0.067 m_{0}$ и $m_{h}=0.45 m_{0}-$ эффективные массы электрона и дырки в GaAs соответственно $[39,40]$ (здесь $m_{0}$ - масса свободного электрона). Фактор $\xi$ выведен в работе [38] из кубических по волновому вектору членов гамильтониана свободных электрона и дырки и имеет вид

$$
\xi=\left[\gamma_{\nu} \frac{m_{h}}{M}\left(a_{1} j_{h}+a_{2} j_{h}^{3}\right)+\gamma_{c} \frac{m_{e}}{M} j_{e}\right]
$$

Здесь $j_{h}$ и $j_{e}-$ значения спинового момента дырки и электрона соответственно; $\gamma_{v}$ и $\gamma_{c}-$ константы при кубических по волновому вектору членах в гамильтонианах свободных дырки и электрона соответственно; $a_{1}$ и $a_{2}-$ безразмерные константы (см. работу [41]). Подставив выражение (1) в (2), мы получим при фиксированном $\omega$ полином от волнового вектора поляритонной волны $K$. Его корни описывают поляритонные дисперсионные зависимости $K_{j}(\omega)$ для
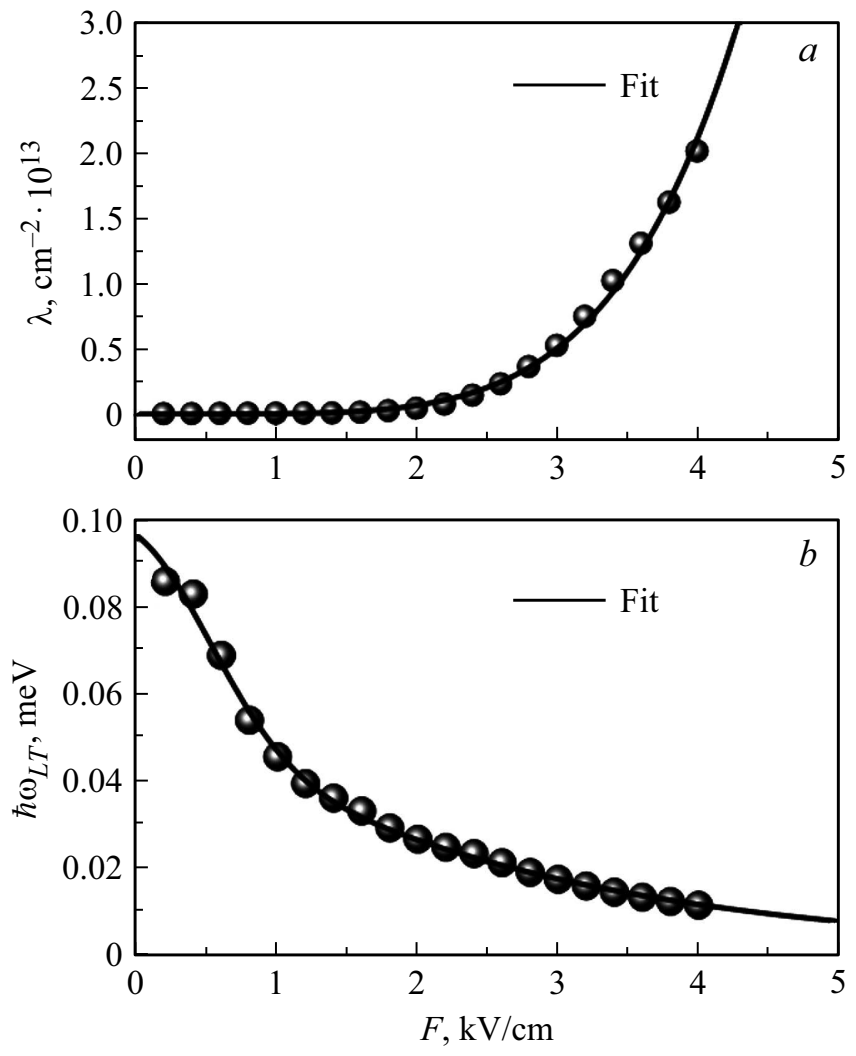

Рис. 1. $a-$ зависимость от поля фактора $\lambda(F)$ (точки), рассчитанная по формуле (4). Сплошная кривая подгонка с функцией $\lambda(F)=a F^{5} \mathrm{c} a=2.1 \cdot 10^{10} \mathrm{\kappa B}^{-5} \cdot \mathrm{cm}^{3}$. $b-$ величина $\hbar \omega_{L T}(F)$ как функция электрического поля (точки). Сплошная кривая - аппроксимация функцией $f=0.049 \exp \left(-1.64 F^{2}\right)+0.0084 F+0.043$.

фотоноподобных и экситоноподобных волн, распространяющихся в прямом и обратном направлениях вдоль оси роста КЯ.

Зависимость энергии экситона от электрического поля описывается фактором $\lambda(F)$ :

$$
\lambda(F)=\frac{1}{\hbar^{2}}\left\langle\phi(F, r)\left|\hat{p}_{x}^{2}-\hat{p}_{y}^{2}\right| \phi(F, r)\right\rangle=\frac{1}{\hbar^{2}}\left(p_{x}^{2}-p_{y}^{2}\right) .
$$

Здесь матричные элементы квадрата импульса относительного движения электрона и дырки, $p_{x}^{2}$ и $p_{y}^{2}$, вычислены на экситонных функциях, $\phi(F, r)$.

Эти матричные элементы и, как следствие, выражение (4) зависят от электрического поля, так как волновая функция относительного движения электрона и дырки $\phi$ зависит от величины $F$. Подробности вычисления представлены в работе [38]. Результаты численного расчета значений $\lambda(F)$ для различных $F$ показаны на рис. $1, a$. Аппроксимация зависимости аналитической функцией приведена в подписи к рисунку.

Заметим, что $\lambda(F) \neq 0$, только если поле имеет ненулевую компоненту в плоскости КЯ. Действительно, при $F_{x}=0$ имеет место цилиндрическая симметрия, так что $p_{x}^{2}=p_{y}^{2}$ и $\lambda(F)=0$ при любом $F$. 
Величина $\hbar \omega_{L T}(F)$ в выражении (2) вычисляется согласно выражению [1]:

$$
\hbar \omega_{L T}(F)=\left(\frac{2 e \hbar P_{1}}{E_{g} m_{0}}\right)^{2} \frac{\pi}{\varepsilon_{0}}|\phi(F, 0)|^{2} .
$$

Здесь $\phi(F, 0)$ - волновая функция, взятая при совпадающих координатах электрона и дырки в экситоне; $\hbar P_{1} / m_{0}=10.3 \cdot 10^{-5}$ мэB $\cdot$ см для GaAs [41]. Так как эта волновая функция зависит от $F$, продольно-поперечное расщепление также зависит от приложенного поля. Зависимость $\hbar \omega_{L T}(\omega)$ сосчитана для различных значений поля и показана на рис. $1, b$. Выражение аппроксимации точек аналитической функцией дано в подписи к рисунку. Детали расчета зависимости $\hbar \omega_{L T}(F)$ можно найти в работе [38].

\section{3. Результаты и обсуждения}

При вычислении спектров электроотражения мы предполагали, что внешний барьерный слой имеет толщину 15 нм и фоновую диэлектрическую проницаемость $\varepsilon_{b 1}=9$. Слой ямы состоит из $\mathrm{GaAs}$, имеет толщину 110 нм, фоновая диэлектрическая проницаемость которого равна $\varepsilon_{0}=12.56$ (см., например, [42]). Диэлектрическая проницаемость полубесконечного барьеpa $\varepsilon_{b 2}=12$, что соответствует материалу AlGaAs. Угол наклона электрического поля к оси $z$ полагали равным $\theta=20^{\circ}$.

Выражения (1)-(2), а также граничные условия Максвелла (МГУ) и дополнительные граничные условия (ДГУ) Пекара $[1,3]$ на гетерограницах позволяют вычислить амплитуды поляритонных волн в КЯ. С помощью МГУ и ДГУ можно связать амплитуды падающей $\left(E_{i}\right)$, прошедшей $\left(E_{t}\right)$ и отраженной $\left(E_{r}\right)$ световых волн с амплитудами поляритонных волн внутри КЯ. Это позволяет вычислить коэффициент отражения света от гетероструктуры с поляритонными резонансами, $R(\omega, F)=\left|E_{r}\right|^{2} /\left|E_{i}\right|^{2}$.

Рис. 2 демонстрирует результаты моделирования спектров электроотражения. $d R(\omega, F) / d F$. Как можно видеть из рисунка, в слабых полях наблюдается несколько десятков спектральных осцилляций, связанных с квантованием движения экситона в КЯ. При увеличении приложенного поля эти осцилляции уменьшаются по амплитуде и полностью исчезают при некотором критическом поле $F_{c} \approx 3.5 \mathrm{\kappa B} / \mathrm{cm}$. При увеличении поля выше критического значения осцилляции вновь возгораются. При этом, спектральное положение максимумов возгоревшихся осцилляций соответствует положению минимумов осцилляций, которые должны наблюдаться при $F<F_{c}$. Это явление мы называем инверсией фазы спектральных осцилляций.

Микроскопическую природу эффекта инверсии спектральных осцилляций поясняет рис. 3. В работах $[25,38]$ показано, что максимумы и минимумы осцилляций полного отражения, связанные с уровнями размерного

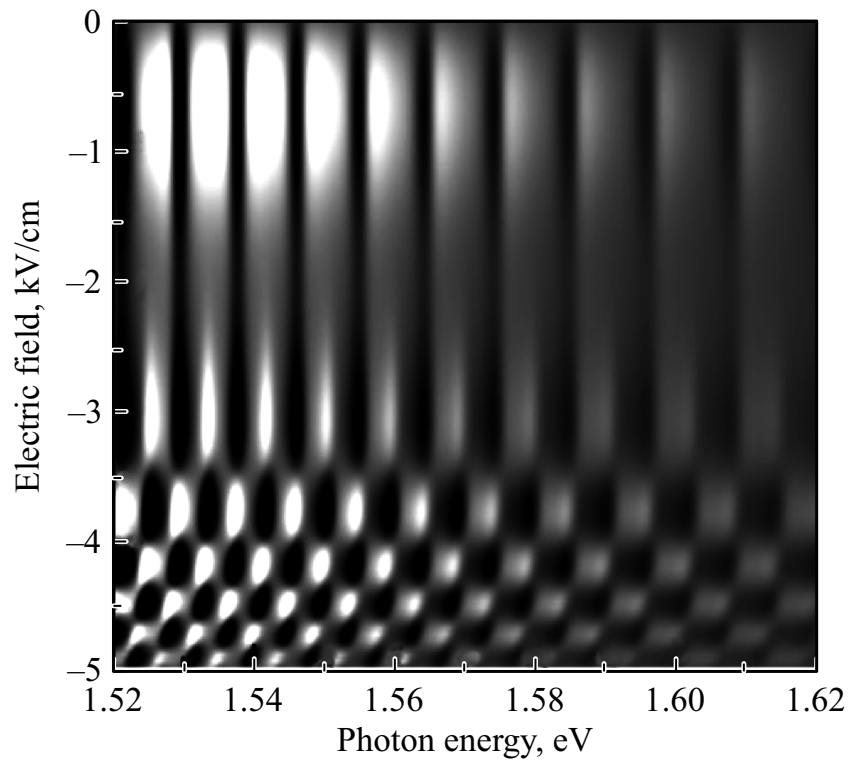

Рис. 2. Электроотражение как функция энергии фотона и приложенного электрического поля, демонстрирующая инверсию фазы спектральных осцилляций.

квантования экситона в квантовой яме, формируются за счет, соответственно, конструктивной и деструктивной интерференции двух каналов переноса энергии (см. рис. 3,a). В первом канале (Ch I) поляритон распространяется в прямом направлении как экситоноподобная волна (с волновым вектором $K_{+}$), а в обратном - как фотоноподобная (с волновым вектором $\left.q_{-}\right)$. При этом в слое КЯ с толщиной $L$ происходит набег фазы, равный $\varphi_{\mathrm{I}}=\left(K_{+}-q_{-}\right) L$. Во втором канале (Ch II) в прямом направлении поляритон распространяется как фотоноподобная волна (с волновым вектором $q_{+}$), а в обратном - как экситонподобная (с волновым вектором $K_{-}$). В этом канале набег фазы равен $\varphi_{\mathrm{II}}=\left(q_{+}-K_{-}\right) L$.

В отсутствие поля $\lambda=0$ и, как это демонстрирует pис. $3, b, K_{+}=-K_{-}$и $q_{+}=-q_{-}$, т. е. распространение поляритонных волн в прямом и обратном направлениях эквивалентно для обеих поляритонных мод, поэтому $\varphi_{\mathrm{I}}=\varphi_{\text {II. }}$ В ненулевом поле линейный по $K$ член в гамильтониане экситона приводит к неэквивалентности распространения экситоноподобной волны в прямом и обратном направлениях, поскольку $\left|K_{+}\right| \neq\left|K_{-}\right|$(см. рис. $3, b)$. При этом для фотоноподобной волны оба направления остаются эквивалентными, т.е. $q_{+}=-q_{-}$. В результате между двумя каналами появляется разность фаз, $\Delta \varphi=\varphi_{\mathrm{I}}-\varphi_{\mathrm{II}}=\left(K_{+}+K_{-}\right) L$, из-за которой условия конструктивной интерференции нарушаются. При монотонном увеличении линейного по $K$ члена эта разность также непрерывно растет. Анализ показывает, что, если $\Delta \varphi=2 \pi N$ ( $N$ - целое число $)$, интерференция конструктивна для уровней размерного квантования с четным порядковым номером и деструктивна для уровней с нечетным порядковым номером. Поэтому в спек- 

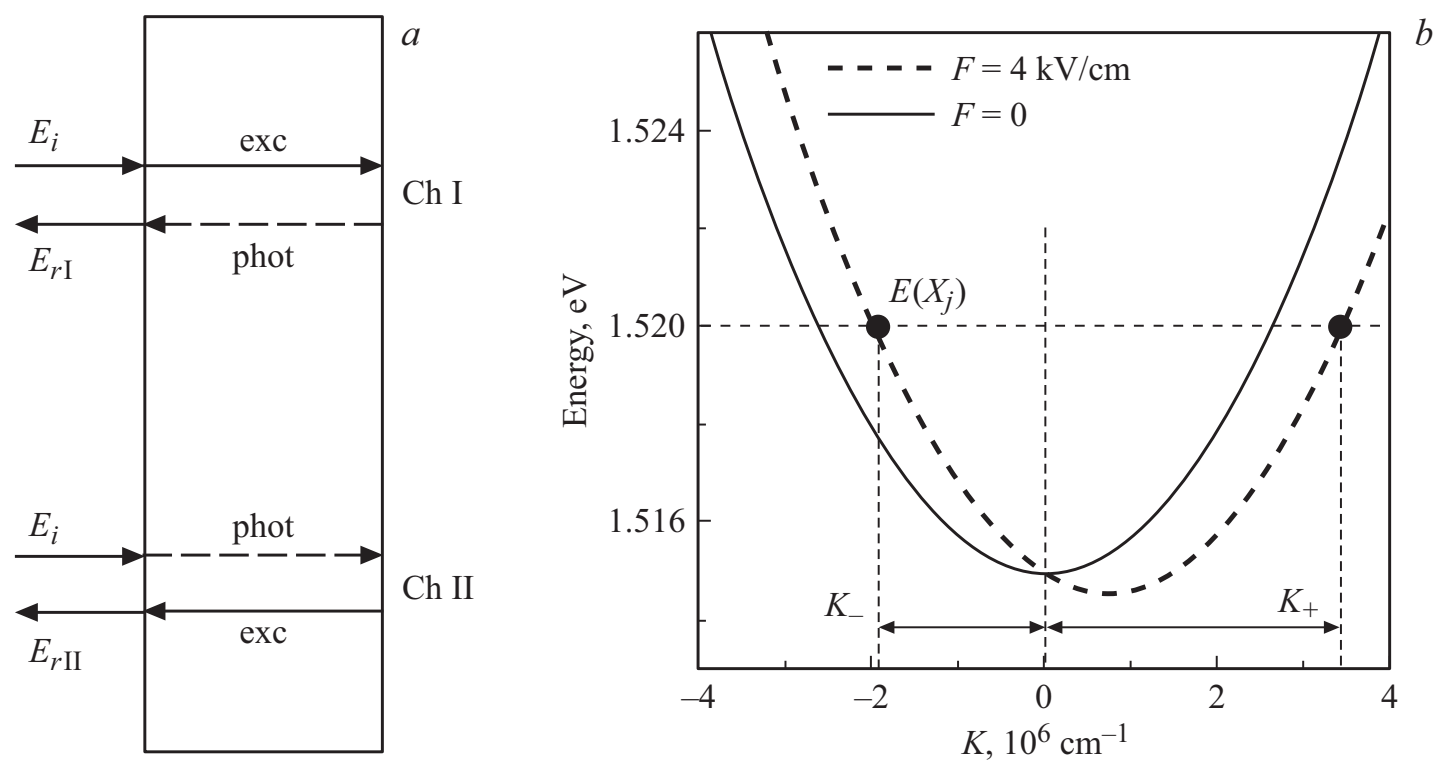

Рис. 3. $a$ - каналы распространения энергии в слое КЯ. Канал Ch I: в прямом направлении вдоль оси роста структуры энергия переносится в экситоноподобной моде (exc) и в обратном направлении - в фотоноподобной моде (phot). Канал Ch II: в прямом направлении энергия переносится в фотоноподобной моде, а в обратном - в экситоноподобной моде. $b-$ дисперсионные зависимости энергии экситона в полях $F=0$ и $F=4$ кВ/см.

трах отражения должны наблюдаться максимумы для четных уровней размерного квантования и минимумы для уровней с нечетным порядковым номером. Если $\Delta \varphi=\pi(N+1 / 2)$, то каналы интерференции Ch I и Ch II полностью гасят друг друга, и осцилляции отражения не наблюдаются. Если $\Delta \varphi=\pi(2 N+1)$, то интерференция конструктивна для нечетных уровней и деструктивна для четных. Благодаря такому периодическому переключению условий конструктивной интерференции для четных и нечетных уровней размерного квантования возможно наблюдение многократной инверсии спектральных особенностей, как это показано на рис. 2. Критические значения электрического поля для каждой инверсии фазы осцилляций становятся все ближе благодаря быстрому росту фактора $\lambda(F)$ с величиной поля (см. рис. $1, a)$.

При анализе спектров мы рассматривали циркулярнополяризованный свет. При этом мы ограничились только одной поляризацией, так как в кросс-поляризации эффект выглядит аналогично. Так как линейную поляризацию можно представить как сумму двух циркулярных, то полученные выводы применимы и к спектрам линейнополяризованного света.

Рассмотрим теперь общие свойства диэлектрического тензора среды с учетом линейного по $K$ члена в экситонном гамильтониане. Диэлектрический тензор, записанный в базисе циркулярно-поляризованных волн, при наличии линейного по $K$ члена имеет простой диагональный вид:

$$
\hat{\varepsilon}(\omega, K)=\left(\begin{array}{cc}
\varepsilon_{+} & 0 \\
0 & \varepsilon_{-}
\end{array}\right),
$$

где

$$
\varepsilon_{ \pm}=\varepsilon_{0}+\frac{\varepsilon_{0} \cdot \hbar \omega_{L T}(F)}{E_{g}-R+\frac{\hbar^{2} K^{2}}{2 M} \pm \lambda(F) \xi K-\hbar \omega+i \hbar \Gamma} .
$$

Здесь знаки „+†“ и „-““ соответствуют поляритонным волнам, имеющим левую и правую циркулярные поляризации соответственно. Тензор (6) диагонален, так как циркулярно-поляризованные волны являются его собственным базисом.

Заметим, что обычно диэлектрический тензор записывают в базисе линейно-поляризованных волн. Если переписать тензор (6) в таком базисе, то он примет вид

$$
\hat{\varepsilon}(\omega, K)=\left(\begin{array}{ll}
\varepsilon_{x x} & \varepsilon_{x y} \\
\varepsilon_{y x} & \varepsilon_{y y}
\end{array}\right) .
$$

Здесь

$$
\begin{aligned}
& \varepsilon_{x x}=\varepsilon_{y y}=\frac{1}{2}\left(\varepsilon_{+}+\varepsilon_{-}\right) \\
& =\varepsilon_{0}+\frac{\varepsilon_{0} \cdot \hbar \omega_{L T}(F)\left(E_{g}-R+\frac{\hbar^{2} K^{2}}{2 M}-\hbar \omega+i \hbar \Gamma\right)}{\left(E_{g}-R+\frac{\hbar^{2} K^{2}}{2 M}-\hbar \omega+i \hbar \Gamma\right)^{2}+(\lambda(F) \xi K)^{2}}, \\
& \varepsilon_{x y}=-\varepsilon_{y x}=\frac{i}{2}\left(\varepsilon_{+}-\varepsilon_{-}\right) \\
& =\frac{i \varepsilon_{0} \hbar \omega_{L T} \lambda(F) \xi K}{\left(E_{g}-R+\frac{\hbar^{2} K^{2}}{2 M}-\hbar \omega+i \hbar \Gamma\right)^{2}-(\lambda(F) \xi K)^{2}}
\end{aligned}
$$

Как можно видеть, в тензоре (8) имеются ненулевые линейные по $K$ недиагональные элементы (10), 
описывающие поворот плоскости поляризации линейнополяризованной световой волны. Среда, характеризуемая таким тензором, является гиротропной [43]. В рассматриваемом нами случае эта гиротропия зависит от приложенного поля через параметр $\lambda(F)$. В отсутствие поля, когда $\lambda=0$, недиагональные элементы тензора становятся равными нулю и гиротропия исчезает.

\section{4. Заключение}

В работе выполнено моделирование спектров электроотражения широкой квантовой ямы, содержащей экситоны, во внешнем электрическом поле, имеющем ненулевую компоненту в плоскости ямы. При монотонном увеличении внешнего электрического поля амплитуда осцилляций электроотражения, связанных с уровнями размерного квантования экситона, может уменьшиться до нуля, после чего вновь возгореться в инвертированной фазе. Показано, что подобная инверсия фазы спектральных осцилляций может происходить многократно. При этом с ростом поля его критические значения, при которых происходит такая инверсия фазы, должны становиться все ближе друг к другу. Анализ экситонного вклада в диэлектрический тензор среды показал, что обсуждаемый в работе эффект является проявлением свойств гиротропии кристалла, которая индуцирована внешним электрическим полем.

\section{Финансирование работы}

Работа поддержана Российским научным фондом, грант № 19-72-20039.

\section{Конфликт интересов}

Авторы заявляют, что у них нет конфликта интересов.

\section{Список литературы}

[1] E.L. Ivchenko. Optical Spectroscopy of Semiconductor Nanostructures (Alpha Science, Harrow, 2005).

[2] C.F. Klingshirn. Semiconductor Optics, 4th ed. (Springer, Berlin, 2012).

[3] A.V. Kavokin, J.J. Baumberg, G. Malpuech, F.P. Laussy. Microcavities (Oxford University, N.Y., 2017).

[4] R. Dingl, W. Wiegmann, C.H. Henry. Phys. Rev. Lett., 33 (14), 827 (1974).

[5] В.А. Киселев, Б.С. Разбирин, И.Н. Уральцев. Письма ЖЭТФ, 18 (8), 504 (1973).

[6] V.A. Kiselev, B.S. Razbirin, I.N. Uraltsev. Phys. Status Solidi B, 72, 161 (1975).

[7] В.А. Киселев, И.В. Макаренко, Б.С. Разбирин, И.Н. Уральцев. ФТТ, 20 (8), 1348 (1977).

[8] A. Tredicucci, Y. Chen, F. Bassani, J. Massies, C. Deparis, G. Neu. Phys. Rev. B, 47, 10348 (1993).

[9] Y. Chen, A. Tredicucci, F. Bassani. Phys. Rev. B, 52 (3), 1800 (1995).
[10] N. Tomassini, A. D’Andrea, R. Del Sole, H. Tuffigo-Ulmer, R.T. Cox. Phys. Rev. B, 51, 5005 (1995).

[11] E. Ubyivovk, Yu.K. Dolgikh, Yu.P. Efimov, S.A. Eliseev, I.Ya. Gerlovin, I.V. Ignatiev, V.V. Petrov, V.V. Ovsyankin. J. Luminesc., 102, 751 (2003).

[12] С.А. Марков, Р.П. Сейсян, В.А. Кособукин. ФТП, 38, 230 (2004).

[13] Д.К. Логинов, Е.В. Убыйвовк, Ю.П. Ефимов, В.В. Петров, С.А. Елисеев, Ю.К. Долгих, И.В. Игнатьев, В.П. Кочерешко, А.В. Селькин. ФТТ, 48, 1979 (2006).

[14] Е.В. Убыйвовк, Д.К. Логинов, И.Я. Герловин, Ю.К. Долгих, Ю.П. Ефимов, С.А. Елисеев, В.В. Петров, О.Ф. Вывенко, А.А. Ситникова, Д.А. Кириленко. ФТТ, 51, 1818 (2009).

[15] A.V. Trifonov, S.N. Korotan, A.S. Kurdyubov, I.Ya. Gerlovin, I.V. Ignatiev, Yu.P. Efimov, S.A. Eliseev, V.V. Petrov, Yu.K. Dolgikh, V.V. Ovsyankin, A.V. Kavokin. Phys. Rev. B, 91, 115307 (2015).

[16] E.S. Khramtsov, P.A. Belov, P.S. Grigoryev, I.V. Ignatiev, S.Yu. Verbin, Yu.P. Efimov, S.A. Eliseev, V.A. Lovtcius, V.V. Petrov, S.L. Yakovlev. J. Appl. Phys., 119, 184301 (2016).

[17] E.S. Khramtsov, P.S. Grigoryev, D.K. Loginov, I.V. Ignatiev, Yu.P. Efimov, S.A. Eliseev, P.Yu. Shapochkin, E.L. Ivchenko, M. Bayer. Phys. Rev. B, 99, 035431 (2019).

[18] J.J. Davies, D. Wolverson, V.P. Kochereshko, A.V. Platonov, R.T. Cox, J. Cibert, H. Mariette, C. Bodin, C. Gourgon, E.V. Ubylvovk, Y.P. Efimov, S.A. Eliseev. Phys. Rev. Lett., 97, 187403 (2006).

[19] A. Litvinov, V.P. Kochereshko, L. Besombes, H. Mariette, D. Loginov, J.J. Davies, L.C. Smith, D. Wolverson. Acta Phys. Polon. A, 112 (2), 161 (2007).

[20] P.S. Grigoryev, V.G. Davydov, S.A. Eliseev, Yu.P. Efimov, V.A. Lovtcius, P.Yu. Shapochkin, I.V. Ignatiev, M. Bayer. Phys. Rev. B, 96, 155404 (2017).

[21] D. Loginov, V.P. Kochereshko, A. Litvinov, L. Besombes, H. Mariette, J.J. Davies, L.C. Smith, D. Wolverson. Acta Phys. Polon. A, 112, 381 (2007).

[22] L.C. Smith, J.J. Davies, D. Wolverson, S. Crampin, R.T. Cox, J. Cibert, H. Mariette, V.P. Kochereshko, M. Wiater, G. Karczewski, T. Wojtowicz. Phys. Rev. B, 78, 085204 (2008).

[23] Д.К. Логинов, В.П. Кочерешко, А.В. Платонов, J.J. Davies, D. Wolverson, L.C. Smith, R.T. Cox, J. Cibert, H. Mariette. ФTT, 51, 1555 (2009).

[24] S.Yu. Bodnar, P.S. Grigoryev, D.K. Loginov, V.G. Davydov, Yu.P. Efimov, S.A. Eliseev, V.A. Lovtcius, E.V. Ubyivovk, V.Yu. Mikhailovskii, I.V. Ignatiev B, 95, 195311 (2017).

[25] D.K. Loginov, A.V. Trifonov, I.V. Ignatiev. Phys. Rev. B, 90, 075306 (2014).

[26] D.K. Loginov, P.S. Grigoryev, Yu.P. Efimov, S.A. Eliseev, V.A. Lovtcius, V.V. Petrov, E.V. Ubyivovk, I.V. Ignatiev. Phys. Status Solidi B, 253, 1537 (2016).

[27] Е.Ф. Гросс. УФН, 76, 433 (1962).

[28] D.F. Blossey. Phys. Rev. B, 2, 3976 (1970).

[29] И.А. Меркулов. ЖЭТФ, 66, 2314 (1974).

[30] F. Bassani, G. Czajkowski, M. Dressler, L. Silvestri. Phys. Status Solidi B, 178, 51 (2000).

[31] Y.-H. Kuo, Y.K. Lee, Y. Ge, Sh. Ren, J.E. Roth, Th.I. Kamins, D.A.B. Miller, J.S. Harris. Nature, 437, 1334 (2005).

[32] B.S. Monozon, P. Schmelcher. Phys. Rev. B, 82, 205313 (2010).

[33] L.V. Butov. Superlat. Microstruct., 108, 2 (2017). 
[34] S.I. Tsintzos, A. Tzimis, G. Stavrinidis, A. Trifonov, Z. Hatzopoulos, J.J. Baumberg, H. Ohadi, P.G. Savvidis. Phys. Rev. Lett., 121, 037401 (2018).

[35] Д.К. Логинов, А.В. Донец. ФТТ, 62, 208 (2020).

[36] Д.К. Логинов, А.В. Донец. ФТТ, 63, 457 (2021).

[37] D.K. Loginov, A.V. Donets. Semiconductors, 52, 562 (2018).

[38] D.K. Loginov, P.A. Belov, V.G. Davydov, I.Ya. Gerlovin, I.V. Ignatiev, A.V. Kavokin, Y. Masumoto. Phys. Rev. Res., 2, 033510 (2020).

[39] M.S. Skolnick, A.K. Jaint, R.A. Stradling, J. Leotinz, J.C. Ousset, S. Askenazy. J. Phys. C, 9, 2809 (1976).

[40] P. Lawaetz. Phys. Rev. B, 4, 3460 (1971).

[41] Г.Е. Пикус, В.А. Марущак, А.Н. Титков. ФТП, 22, 185 (1988).

[42] G.E. Stillman, D.M. Larsen, C.M. Wolfe, R.C. Brandt. Solid State Commun., 9, 2245 (1971).

[43] В.М. Агранович, В.Л. Гинзбург. Кристаллооптика с учетом пространственной дисперсии и теория экситонов (М., Наука, 1979).

Редактор А.Н. Смирнов

\section{Influence of an electric field on the exciton moving in GaAs}

D.K. Loginov, P.A. Belov, I.Ya. Gerlovin, I.V. Ignatiev

St. Petersburg State University, Spin Optics Laboratory,

198504 Petrodvorets, St. Petersburg, Russia

Abstract A theoretical modeling of the interference of polaritonic waves propagating across a quantum well in the presence of a tilted electric field is performed. It is demonstrated that the interference patterns are constructive and destructive at different strengths of electric field. This phenomenon is described by the linear-in-K term in the exciton Hamiltonian. 